\title{
Alzheimer's disease: the controversial approval of Aducanumab
}

\author{
Fabrizio Tagliavini ${ }^{1} \cdot$ Pietro Tiraboschi $^{1} \cdot$ Antonio Federico $^{2}$
}

Accepted: 12 July 2021 / Published online: 29 July 2021

(c) Fondazione Società Italiana di Neurologia 2021

\begin{abstract}
Alzheimer's disease (AD) is the most common form of dementia, affecting millions of people worldwide and causing major personal and family distress and socio-economic burden. The disease is distinguished by misfolding and aggregation of amyloid $\beta$ (A $\beta$ ) in the neuropil (senile plaques) and vessel walls (congophilic angiopathy), and by hyperphosphrylation, misfolding, and assembly of microtubule-associate protein Tau into abnormal filaments within nerve cell bodies and processes (neurofibrillary tangles). These events are associated with neuroinflammation and lead to neuronal degeneration and progressive cognitive impairment. Based on the observation that the rare genetic forms of the disease $(<1 \%$ of all cases) are linked to mutations in the $\mathrm{A} \beta$ precursor protein, presenilin 1 , or presenilin 2 genes resulting in increased production and/or aggregation properties of $\mathrm{A} \beta$, it has been advanced that $\mathrm{A} \beta$ pathology is the trigger of the process and, consequently, the primary therapeutic target [1]. Accordingly, strategies aimed at removing or preventing $A \beta$ accumulation have been proposed, in particular immunotherapy with monoclonal antibodies against $\mathrm{A} \beta$. However, trials in sporadic AD were mostly disappointing since they failed to demonstrate clinical benefits, although many were effective in clearing $A \beta$ deposits [2].
\end{abstract}

On June 7, the US Food and Drug Administration (FDA) approved the monoclonal antibody Aducanumab for the treatment of Alzheimer's disease [3]. Following the results of a double-blind, placebo-controlled phase $1 \mathrm{~b}$ randomized trial [4], the drug was used in two phase III clinical trials that were halted for futility after an interim analysis. However, a re-evaluation of the studies on a larger set of data available after the trials were discontinued showed that in one of the

Antonio Federico

federico@unisi.it

1 Fondazione IRCCS Istituto Neurologico Carlo Besta, 20133 Milan, Italy

2 Department Medicine, Surgery and Neurosciences, Medical School, University of Siena, Viale Bracci 2, 53100 Siena, Italy two trials (but not in the other), treatment with Aducanumab at the highest dose $(10 \mathrm{mg} / \mathrm{kg})$ resulted in a $23 \%$ reduction in clinical decline compared to placebo, as determined by the Clinical Dementia Rating scale Sum of Boxes, a composite measure with cognitive and functional components that was set as the primary efficacy endpoint [5]. Given the negative results of the other study that did not meet the clinical endpoint and the high incidence of adverse events with the effective dose, in particular amyloid-related imaging abnormalities [5], this decision was considered questionable and premature by the FDA Nervous System Drug Advisory Committee, and three of the 11 members resigned a few days after approval. This has triggered a broad debate in the scientific community [6, 7].

The path used by FDA for Aducanumab was the accelerated approval program which allows early approval of drugs for severe conditions for which there are no effective therapies. Approval was based on a surrogate clinical endpoint, namely the reduction of amyloid plaques in the brain, in the assumption that this is likely to translate into a clinical benefit to patients.

However, the scientific community does not entirely agree on the "A $\beta$ cascade hypothesis" and the therapeutic effectiveness of removing amyloid plaques. Autopsy studies of patients subjected to active immunization showed that clearance of amyloid deposits neither halted nor slowed the progression of the disease, not affecting tau propagation and loss of synapses and neurons [8]. Following these controversies, the FDA did not grant the medication full approval. The agency ordered the drug's makers to conduct a postmarketing phase IV study over the next 9 years to confirm the drug positive effect on cognitive deterioration. If clinical efficacy is not documented, approval will be withdrawn.

To note is that Aducanumab has been approved for patients with Alzheimer's disease without specifying whether demonstration of A $\beta$ pathology by CSF analysis or PET scan is mandatory [9], a surprising aspect as patient inclusion in the trial and drug approval were based on the presence and subsequent removal of amyloid plaques. 
It is to be expected that the approval of the drug will have a number of consequences.

First, if removal of amyloid $\beta$ as a surrogate end-point is sufficient for approval, clinical outcome measures may not be mandatory in future studies, and other pharma companies developing monoclonal antibodies that have not been approved due to failure to meet primary clinical end-points may now require it. Second, since a drug is now available, the attrition rate of potential volunteer participants to new drug trial and the number of available subjects may decrease, and the recruitment time and the drop-out rate may increase. Third, this smoother, fast-track approval path represents a regulatory precedent that could eventually be extended to drug candidates for other neurodegenerative diseases for which there are no effective treatments.

These are just some of the issues that could impact on the development of other drugs that may be needed for a successful treatment of this devastating disorder, given its complexity related to many molecular players and pathways involved, which are likely to have different relevance at different stages of the disease.

The approval of the first potentially disease-modifying drug for Alzheimer's disease has given hope and new strength to patients and families, who had been waiting for a step forward in treatment since 2003, when the last drug was approved. It has also renewed the interest of big pharma and investors in drug development for neurodegenerative disorders, which had been waning due to many failures. This positive aspect will hopefully give new impetus to research aimed at developing additional therapeutic strategies and finding clinically effective treatments.

However, the scientific community should have a prudential attitude in front of these very fast approval processes, waiting for consistent and conclusive results, also in relation to the high cost of a treatment that must be clearly related to its effectiveness. As Europeans, we need the position of the EMA. As neuroscientists, we have to gain fully insight into the factors that govern the development and progression of the pathological process. As neurologists, we have to prepare for a deeper understanding of the clinical aspects of the disease, and to improve the use of different biomarkers and the ability to better correlate them to clinical findings.

\section{Declarations}

Conflict of interest The authors declare no competing interests.

Ethical approval None.

\section{References}

1. Hardy JA, Higgins GA (1992) Alzheimer's disease: the amyloid cascade hypothesis. Science 256(5054):184-185. https://doi.org/ 10.1126/science. 1566067

2. Panza F, Lozupone M, Logroscino G, Imbimbo BP (2019) A critical appraisal of amyloid-beta-targeting therapies for Alzheimer disease. Nat Rev Neurol 15:73-88. https://doi.org/10.1038/ s41582-018-0116-6

3. FDA. FDA's decision to approve new treatment for Alzheimer's disease. 2021 Jul 6 [cited 2021 Jun 14]. Available from: https:// www.fda.gov/drugs/news-events-human-drugs/fdas-decisionapprove-new-treatment-alzheimers-disease. Accessed 21 Jul 2021

4. Sevigny $\mathrm{J}$, Chiao $\mathrm{P}$, Bussière $\mathrm{T}$, Weinreb $\mathrm{PH}$, Williams L et al (2016) The antibody aducanumab reduces $A \beta$ plaques in Alzheimer's disease. Nature 537(7618):50-56. https://doi.org/10.1038/ nature 19323

5. Biogen (2019) EMERGE and EMERGE and ENGAGE topline results: two phase 3 studies to evaluate aducanumab in patients with early Alzheimer's disease. Available from: https://investors. biogen.com/static-files/ddd45672-9c7e-4c99-8a06-3b557697c0 6f. Accessed $21 \mathrm{Jul} 2021$

6. Perlmutter JS (2021) FDA's green light, science's red light. Science 372(6549):1371. https://doi.org/10.1126/science.abk0575

7. Mullard A (2021) Controversial Alzheimer's drug approval could affect other diseases. Nature 595(7866):162-163. https://doi.org/ 10.1038/d41586-021-01763-9

8. Nicoll JAR, Buckland GR, Harrison CH, Page A, Harris S, Love S, Neal JW, Holmes C, Boche D (2019) Persistent neuropathological effects 14 years following amyloid- $\beta$ immunization in Alzheimer's disease. Brain 142(7):2113-2126. https://doi.org/10.1093/ brain/awz142

9. FDA. Prescribing Information for ADUHELM. Revised July 2021. Available from: https://www.accessdata.fda.gov/drugsatfda_docs/ label/2021/761178s0031bl.pdf. Accessed 21 Jul 2021

Publisher's Note Springer Nature remains neutral with regard to jurisdictional claims in published maps and institutional affiliations. 